DOSSIER

\title{
Évaluation d'entreprises
}

Évolution des méthodes et contextes

sous la direction de

Pierre Astolfi Édouard Chastenet

Alain Marion Arnaud Thauvron 
PIERRE ASTOLFI

$I A E$, Gustave Eiffel

ÉDOUARD CHASTENET

$I A E$, Université Lyon 3

\section{ALAIN MARION}

Université Lyon 3

\section{ARNAUD THAUVRON}

$I A E$, Université Paris-Est

\section{Méthode des multiples}

\section{Contribution à l'évaluation des entreprises}

La méthode des multiples en évaluation d'entreprise est sans doute l'une des approches les plus couramment utilisées par les praticiens. Pour autant, compte tenu de ses limites, la méthode suscite un certain nombre de questions dès lors qu'il s'agit de la mettre en œuvre. Dans ce contexte, les auteurs proposent, en s'appuyant sur la mesure du taux d'erreur absolu de l'évaluation, un mode opératoire permettant d'améliorer la pertinence de la méthode. Les résultats de leurs travaux conduisent notamment à privilégier l'utilisation de multiples fondés sur des résultats prévisionnels (en l'occurrence I'Ebitda ou l'Ebit). Par ailleurs, ils montrent que le processus de sélection de sociétés comparables peut être amélioré en utilisant des ratios de performance, et en particulier le taux de croissance attendu des résultats. 
$\mathrm{L}$ es banques d'affaires, les directions financières, l'administration fiscale, les analystes financiers ainsi que de nombreux professionnels concernés par l'évaluation d'entreprise sont régulièrement sollicités pour estimer la valeur des entreprises, cotées ou non, dans différents contextes : boursier, transactionnel, comptable, juridique, fiscal...

En raison de sa simplicité d'usage, la méthode des multiples, notamment lorsqu'elle se fonde sur la valeur boursière de sociétés " comparables », est l'une des méthodes d'évaluation des entreprises les plus appliquées par les professionnels, à titre principal ou en complément de la méthode des flux de trésorerie actualisés (Discounted Cash-Flows, DCF) (Liu et al., 2007).

En revanche, la méthode est souvent considérée comme trop simple pour pouvoir prendre en compte l'ensemble des caractéristiques des entreprises, notamment celles qui les différencient des sociétés auxquelles elles sont comparées (Suozzo et al., 2001), et trop subjective quant au choix des multiples « appropriés » (Damodaran, 2006). Cela conduit Crow et al. (2001) à considérer, dans un propos quelque peu provocateur, que la méthode des multiples serait la méthode la plus mal comprise et la plus mal appliquée par les professionnels.

Cette popularité et les critiques formulées à son encontre sont à l'origine de l'intérêt grandissant que lui porte la recherche académique depuis la fin des années 1990 : la littérature s'intéresse plus particulièrement aux critères de sélection des multiples ou des sociétés comparables à retenir pour améliorer la pertinence de la méthode, l'objectif étant de réduire l'écart entre la valeur de l'entreprise effectivement obser- vée sur le marché et sa valeur estimée par application de la méthode.

Après un état des lieux des pratiques, nous rappelons que la méthode des multiples présente un certain nombre de limites. Il est notamment possible de s'interroger sur la qualité des données sources nécessaires aux calculs des multiples de valorisation, qu'il s'agisse des indicateurs figurant dans les états financiers publiés en application des normes comptables ou des indicateurs financiers prévisionnels qui sont issus des consensus d'analystes financiers

Ces réserves nous conduisent à déployer une démarche méthodologique fondée sur une étude empirique venant confirmer et compléter les résultats des recherches antérieures.

Nos résultats, illustrés par un cas d'application, permettent de formuler des propositions opératoires de nature à améliorer l'efficience du processus d'évaluation des entreprises.

\section{I - PRATIQUES ET LIMITES DE LA MÉTHODE DES MULTIPLES}

\section{1. État des lieux des pratiques}

Parmi les différentes approches à la disposition des évaluateurs, la méthode des multiples est fréquemment utilisée par les praticiens dans la mesure où elle présente de nombreux avantages, en particulier sa simplicité. La méthode consiste, dans un premier temps, à constituer un échantillon de sociétés cotées « comparables » à l'entreprise à évaluer (appartenant, a minima, au même secteur d'activité) et à calculer, pour chacune d'entre-elles, un ou plusieurs multiples représentatifs de leur niveau de valorisation, obtenus en rapportant leur valeur observée sur le marché (capitalisa- 
tion boursière, valeur d'entreprise ${ }^{1}$ ou prix de transaction ${ }^{2}$ ) à un inducteur de valeur, le plus souvent un indicateur comptable ou financier représentatif de leur performance actuelle ou future (chiffre d'affaires, résultat d'exploitation, résultat net ajusté...).

Il convient ensuite d'appliquer ces multiples aux indicateurs comptables ou financiers de l'entreprise à évaluer pour obtenir une estimation rapide de sa valeur ou valider les résultats d'une approche de valorisation plus complexe, fondée sur la méthode des DCF par exemple (Liu et al., 2007).

Les praticiens mettent souvent en avant le fait que la méthode des multiples est facile à mettre en œuvre car elle nécessite moins d'hypothèses à formuler et moins d'informations à traiter que d'autres méthodes fondées sur l'analyse fondamentale, telle la méthode des DCF (Damodaran, 2006). De plus, la méthode ne nécessite pas d'estimer un taux d'actualisation, tel que le coût du capital, ni de réaliser une analyse approfondie du modèle économique de l'entreprise. Enfin, les données utilisées sont facilement accessibles à partir de bases de données comptables et financières. Courteau et al. (2006) suggèrent ainsi que les analystes financiers ont recours à des multiples de valorisation quand ils sont dans l'incertitude quant aux éléments à prendre en compte au-delà de l'horizon prévisionnel

Tableau 1 - Méthodes d'évaluation et multiples les plus utilisés par les analystes financiers

\begin{tabular}{|c|c|c|c|c|}
\hline & \multicolumn{4}{|c|}{ Auteurs } \\
\hline & $\begin{array}{l}\text { Chastenet et } \\
\text { Jeannin (2007) }\end{array}$ & $\begin{array}{l}\text { Bellier Delienne } \\
\text { et Bour (2007) }\end{array}$ & $\begin{array}{c}\text { Harbula } \\
(2009)\end{array}$ & $\begin{array}{c}\text { Bancel et } \\
\text { Mittoo (2013) }\end{array}$ \\
\hline Période d'observation & 2005 & 2005-2006 & 2002-2006 & 2012 \\
\hline Méthode des DCF & $85 \%$ & $71 \%$ & $70 \%$ & $80 \%$ \\
\hline $\begin{array}{l}\text { Méthode des } \\
\text { multiples }\end{array}$ & $71 \%$ & $79 \%$ & $80 \%$ & $80 \%$ \\
\hline $\begin{array}{c}\text { PER } \\
\text { (Price Earning Ratio) }\end{array}$ & $89 \%$ & $58 \%$ & $80 \%$ & $68 \%$ \\
\hline $\begin{array}{c}\text { PTB } \\
\text { (Price To Book) }\end{array}$ & $6 \%$ & $\mathrm{n} / \mathrm{r}$ & $50 \%$ & $47 \%$ \\
\hline VE/Chiffre d'affaires & $50 \%$ & $42 \%$ & $50 \%$ & $\mathrm{n} / \mathrm{r}$ \\
\hline VE/Ebitda ${ }^{3}$ & $55 \%$ & $55 \%$ & $75 \%$ & $83 \%$ \\
\hline VE/Ebit ${ }^{4}$ & $41 \%$ & $57 \%$ & $70 \%$ & $46 \%$ \\
\hline
\end{tabular}

1. Valeur d'entreprise $=$ capitalisation boursière de la société + valeur de marché de ses dettes financières nettes.

2. Prix auquel a été valorisée la société à l'occasion d'un transfert de contrôle.

3. L'Ebitda (Earning Before Interest, Tax, Depreciation and Amortization) correspond approximativement à l'excédent brut d'exploitation défini par le PCG (après déduction de la participation des salariés).

4. L'Ebit (Earning Before Interest and Tax) correspond au résultat opérationnel défini par les normes comptables internationales. 
retenu. Sur un autre plan, il peut aussi être mis en avant le fait que la méthode est aisée à expliquer et à présenter à des interlocuteurs non avertis (DeAngelo, 1990).

Comme le montre le tableau 1, qui présente une synthèse des études consacrées aux méthodes d'évaluation et aux multiples utilisés par les analystes financiers, la méthode des multiples est d'utilisation aussi fréquente que celle des DCF, avec un recours principal au PER (multiple correspondant au rapport entre le cours et le bénéfice par action) et, sur une période plus récente, au multiple de l'Ebitda. Dans une moindre mesure, les autres inducteurs de valeurs utilisés sont l'Ebit, le chiffre d'affaires ou les capitaux propres comptables (avec le Price To Book).

\section{Limites de la méthode}

D'un point de vue théorique, s'il est possible de définir analytiquement des inducteurs de valeur qui expliquent le niveau de valorisation des sociétés cotées, leurs multiples de valorisation sont alors exploitables pour évaluer d'autres entreprises, cotées ou non, sous réserve qu'elles leurs soient comparables par le profil et le risque des flux de trésorerie qu'elles sont susceptibles de générer dans le futur (Liu et al., 2002). La mise en œuvre de la méthode des multiples repose ainsi sur deux étapes principales (Holthausen et Zmijewski, 2012), à savoir : - la constitution d'un échantillon d'entreprises comparables ;

- l'identification des inducteurs de valeur (indicateurs comptables ou financiers les plus représentatifs de la valeur de l'entreprise).

La démarche repose sur un certain nombre d'hypothèses qui constituent autant de faiblesses de la méthode des multiples, sus- ceptibles de générer des erreurs ou de laisser la place à des manipulations si l'évaluateur ne s'appuie pas sur une méthodologie rigoureuse.

Rappelons que la méthode des multiples est fondée sur le concept de «valeur de marché » qui présuppose que le marché est suffisamment efficient, en moyenne au moins, pour attribuer une valeur similaire à des actifs également similaires (Esty, 2000 ; Meitner, 2006). Dans le cas contraire, des opportunités d'arbitrages existeraient. Deux entreprises identiques doivent donc avoir la même valeur.

Dans la pratique, les évaluateurs se fondent généralement sur la constitution de « groupes de pairs sectoriels » (industry peer groups), en sélectionnant des sociétés, a priori considérées comme « comparables » en raison de leur appartenance au même secteur d'activité que l'entreprise à évaluer. Cette approche relativement simple conduit à supposer que toutes les entreprises d'un même secteur d'activité ont les mêmes caractéristiques en termes de risques et de perspectives de flux de trésorerie futurs, ce qui est loin de correspondre à la réalité (Damodaran, 2006).

Dans un second temps, les évaluateurs doivent sélectionner les inducteurs de valeur les plus pertinents pour évaluer l'entreprise. Compte tenu des fondements théoriques de tout modèle d'évaluation, qu'il s'agisse de la méthode des DCF ou de la méthode des multiples, il convient d'identifier des indicateurs comptables ou financiers qui traduisent le mieux la capacité de l'entreprise à générer des flux de trésorerie récurrents dans le futur.

Dans cette optique, le recours à des indicateurs strictement comptables (qu'il s'agisse 
du résultat net, du résultat d'exploitation ou du chiffre d'affaires directement issus des états financiers, sans aucun ajustement) revient à considérer que ces derniers sont en capacité de fournir une information pertinente quant à la performance future de l'entreprise et présentent, de ce fait, les qualités requises pour l'évaluation de

\section{PERTINENCE DES INDICATEURS COMPTABLES MIS À DISPOSITION DES ÉVALUATEURS}

La qualité des indicateurs comptables utilisés pour le calcul des multiples est contestée, tant par les émetteurs que par les utilisateurs. En effet, ces indicateurs sont issus des états financiers publiés en application des normes comptables dont la pertinence est remise en cause par de nombreuses parties prenantes.

Selon Kothari et al. (2010), les états financiers établis en application de ces normes (US GAAP et IFRS) ne seraient pas en mesure de faciliter les travaux d'évaluation. En effet, selon leur vision originale, les objectifs assignés à ces états financiers en termes de réduction de l'asymétrie d'information à destination des investisseurs, d'une part, et en termes d'évaluation des capitaux propres en « juste valeur », d'autre part, loin de se compléter, ont au contraire tendance à s'opposer.

Pour Casta (2003), la question de la légitimité des normes, appréciée à la lumière de leur capacité à apporter une réponse pertinente à une demande effectivement exprimée par les utilisateurs, est également au cœur de la réflexion liée au concept de valeur. Ce dernier souligne d'ailleurs que « la promotion du modèle d'évaluation à la juste valeur relève davantage d'une logique de l'offre que d'une demande explicite de la part des utilisateurs ».

Pour sa part, Nobes (2006) souligne l'inertie des émetteurs qui peuvent souhaiter maintenir des pratiques comptables antérieures à l'adoption des normes IAS/IFRS, dans le but de perturber le moins possible leur comptabilité en vue de favoriser une meilleure compréhension tant interne qu'externe de l'information produite.

La capacité des normes comptables internationales à fournir des données directement utiles aux investisseurs pour évaluer les entreprises est donc remise en cause par un certain nombre de facteurs qui conduisent les évaluateurs, pour appliquer la méthode des multiples, à retenir des indicateurs financiers " ajustés ou absents des comptes », c'est-à-dire des indicateurs qui ne sont pas définis par les normes comptables (tels que l'Ebitda). Constatant que les émetteurs publient ce type d'indicateurs, parce qu'ils les jugent pertinents ou parce qu'ils répondent à une attente des investisseurs ou des analystes, l'Autorité des marchés financiers (AMF), à l'instar de l'European Securities and Markets Authority (ESMA) au niveau européen, a publié en $2010^{5}$ une recommandation les invitant notamment à définir ces indicateurs, ajustés ou absents des compte, de façon précise et à les rapprocher des données comptables publiées.

5. Recommandation AMF n 2010-11 du 17 novembre 2010 relative à la communication des sociétés sur leurs indicateurs financiers. 
l'entreprise. La recherche en comptabilité montre la nécessité de nuancer cette hypothèse (cf. encadré : «Pertinence des indicateurs comptables mis à disposition des évaluateurs $»)$.

Par ailleurs, le recours à des indicateurs financiers prévisionnels issus de consensus d'analystes suppose que les prévisions de ces derniers soient suffisamment fiables. En la matière, un horizon prévisionnel de deux ans est sans doute le plus adapté, comme le montre la pratique la plus courante des analystes financiers (Penman, 2006). Rappelons que les principaux consensus d'analystes disponibles dans les bases de données financières délivrent des prévisions portant sur le résultat net par action, d'une part, et sur le chiffre d'affaires, l'Ebitda et l'Ebit, d'autre part.

Outre l'utilisation de ces indicateurs comptables ou financiers comme inducteurs de valeur (pour le calcul des multiples de valorisation), ceux-ci sont aussi susceptibles d'être pris en compte pour affiner le périmètre des sociétés comparables. Papelu et al. (2000) considèrent que, pour être réellement comparables, les sociétés doivent avoir les mêmes caractéristiques opérationnelles et financières. Dans cette optique, il est préconisé de tenir compte de ratios de performance, tels que le taux de rentabilité (soit le rapport entre l'Ebit et les capitaux employés de l'entreprise) ou le taux de croissance attendu des résultats pour sélectionner les multiples des sociétés les plus comparables au sein d'un groupe de pairs sectoriels (Bhojraj et Lee, 2002 ; Hermann et Richter, 2003).

\section{II - DÉMARCHE PERMETTANT D'AMÉLIORER LE PROCESSUS D'ÉVALUATION}

\section{Présentation de la démarche}

L'un des objectifs de notre étude est d'identifier les critères les plus pertinents pour sélectionner les multiples et les entreprises comparables, cela afin de limiter les risques d'erreur, voire de manipulation des valeurs calculées. L'objectif final est de minimiser l'écart entre la valeur estimée par la méthode des multiples et la valeur telle qu'elle peut être observée sur le marché quand l'entreprise est cotée.

Notre recherche suit les différentes étapes devant être mises en œuvre pour appliquer la méthode :

- constitution d'un échantillon de sociétés cotées appartenant au même secteur d'activité que l'entreprise à évaluer ${ }^{6}$ (dit groupe de pairs sectoriels), le cas échéant regroupées en fonction de certains ratios de performance (taux de rentabilité, taux de marge ou taux de croissance, etc.) afin d'identifier les sociétés qui lui sont les plus comparables; - calcul des multiples de valorisation de chacune de ces sociétés, correspondant au rapport entre leur valeur marché et les différents indicateurs comptables ou financiers représentatifs de leur performance (chiffre d'affaires, résultat d'exploitation ou résultat net réalisés, en cours ou prévisionnels, indicateurs correspondant aux consensus des analystes financiers) ;

- calcul des multiples «synthétiques » représentatifs du niveau de valorisation des sociétés constituant l'échantillon, corres-

6. Entreprise elle-même cotée dans le contexte de la présente étude. 


\section{MÉTHODOLOGIE DE LA RECHERCHE}

La pertinence d'un multiple dépend de sa capacité à réduire le taux d'erreur de l'évaluation, à savoir la capacité de ce multiple à fournir une estimation de la valeur de l'entreprise que l'on cherche à évaluer aussi proche que possible de sa valeur de marché.

La pertinence est appréciée à partir d'un échantillon d'entreprises cotées comprenant 919 à 1192 sociétés européennes observées sur la période 2006-2008, répertoriées par la base de données FactSet et regroupées au sein de 59 à 70 groupes de pairs sectoriels selon la nomenclature de niveau 2 établie par cette base (cf. tableau 2).

Pour chaque entreprise évaluée, et pour chaque multiple utilisé, l'erreur de l'évaluation est donnée par l'équation suivante :

où :

$$
\varepsilon_{j}^{x}=\operatorname{Vobs}_{j}-\text { Vest }_{j}^{x} \text { avec Vest } t_{j}^{x}=\text { Mest }^{x} \times x_{j}
$$

- Vobs ${ }_{\mathrm{j}}$ correspond à la valeur de marché de l'entreprise $\mathrm{j}$ observée à la date d'évaluation, - $x_{j}$ à l'indicateur comptable ou financier de l'entreprise $\mathrm{j}$, disponible à la date de l'évaluation,

- Mest ${ }^{\mathrm{x}}$ au multiple sectoriel estimé et appliqué à l'indicateur $\mathrm{x}_{\mathrm{j}} \mathrm{à} \mathrm{la} \mathrm{date} \mathrm{d'évaluation,} \mathrm{à}$ savoir le multiple synthétique le plus représentatif du niveau de valorisation des sociétés constituant le groupe de pairs sectoriels, éventuellement regroupées selon certains ratios de performance afin d'identifier celles qui sont le plus comparables à l'entreprise $\mathrm{j}$,

- Vest ${ }_{j}^{\mathrm{x}}$ à la valeur de l'entreprise $\mathrm{j}$ estimée à la date d'évaluation par application du multiple Mest $^{\mathrm{x}}$ à l'indicateur $\mathrm{x}_{\mathrm{j}}$, et

$-\varepsilon_{j}^{\mathrm{x}}$ à l'erreur d'évaluation de l'entreprise $\mathrm{j}$ résultant de l'application de ce multiple ${ }^{7}$.

Pour mémoire, les multiples synthétiques représentatifs du niveau de valorisation d'un groupe de pairs sectoriels, à savoir un échantillon de n sociétés regroupées selon leur secteur d'activité et, le cas échéant, selon certains ratios de performance, sont calculés selon les formules suivantes ${ }^{8}$ :

- Multiple synthétique correspondant à la moyenne arithmétique :

$$
\text { Mest }^{x}=\left(\sum_{i=1}^{n} \operatorname{Vobs}_{i} / x_{i}\right) / n
$$

- Multiple synthétique correspondant à la moyenne harmonique :

$$
\text { Mest }^{x}=n \times\left(\sum_{i=1}^{n} x_{i} / \operatorname{Vobs}_{i}\right)
$$

7. L'ensemble des données comptables utilisées dans le cadre de cette étude pour le calcul de la dette nette et des capitaux employés sont issues de la base de données FactSet. Les indicateurs financiers d'exploitation (chiffre d'affaires, Ebitda et Ebit), qu'ils soient relatifs aux exercices réalisés, en cours et prévisionnels, sont issus du consensus d'analystes financiers réalisé par FactSet.

8. Les multiples synthétiques généralement calculés par les praticiens correspondent aux multiples moyens ou médians des sociétés constituant un groupe de pairs sectoriels. Il est toutefois démontré que le multiple qui minimise l'erreur de l'évaluation correspond à la moyenne harmonique des multiples d'un échantillon (cf. Liu et al., 2002). 
Le taux d'erreur absolu (TEA) de l'évaluation, pour chaque entreprise $\mathrm{j}$, et pour chaque multiple appliqué, est quant à lui calculé selon la formule suivante :

$$
T E A_{j}^{x}=\left|\frac{V o b s_{j}-V e s t_{j}^{x}}{V o b s_{j}}\right|=\left|\frac{\varepsilon_{j}^{x}}{V o b s_{j}}\right|
$$

Pour un groupe de pairs sectoriels ou un échantillon d'entreprises, comparer la pertinence des multiples revient à comparer le TEA de l'évaluation qui résulte de leur application, suivant en cela la méthodologie la plus répandue dans la littérature (Liu et al., 2002 et 2007 ; Dittmann et Weiner, 2005 ; Yoo, 2006 ; Schreiner et Spremann, 2007). Les tests statistiques réalisés portent sur la comparaison des TEA de l'évaluation résultant de l'application de chacun des multiples calculés (dans le cas de notre étude, nous avons eu recours à des tests paramétriques fondés sur la comparaison des moyennes, des variances ou des proportions).

Tableau 2 - Nombre de sociétés de l'échantillon par date d'observation ${ }^{9}$

\begin{tabular}{|c|c|c|c|c|}
\hline Date d'observation & Sociétés & Pays & Secteurs - niveau 1 & Secteurs - niveau 2 \\
\hline Population & $\mathbf{6 ~ 0 6 8}$ & $\mathbf{3 3}$ & $\mathbf{1 8}$ & $\mathbf{1 1 2}$ \\
\hline 31 mars 2006 & 919 & 24 & 17 & 59 \\
\hline 31 mars 2007 & 1090 & 24 & 18 & 66 \\
\hline 31 mars 2008 & 1192 & 27 & 18 & 70 \\
\hline
\end{tabular}

pondant à la médiane ou à la moyenne de chacun de ces multiples ;

- estimation de la valeur de l'entreprise à évaluer par application de ces multiples synthétiques.

À l'instar de la plupart des études académiques, cette démarche est complétée par une analyse, souvent omise par les praticiens, consistant à mesurer le taux d'erreur de l'évaluation, correspondant, pour chaque entreprise évaluée, à la différence entre sa valeur estimée par application des multiples synthétiques calculés et sa valeur observée sur le marché.

Nos travaux confirment et complètent les résultats des études empiriques publiées au cours des quinze dernières années en ce qui concerne, notamment :

- l'identification des indicateurs comptables ou financiers les plus pertinents pour calculer les multiples pour chacun des groupes de pairs sectoriels constitués (cf.

9. L'ensemble des données comptables utilisées dans le cadre de cette étude pour le calcul de la dette nette et des capitaux employés sont issues de la base de données FactSet. Les indicateurs financiers d'exploitation (chiffre d'affaires, Ebitda et Ebit), qu'ils soient relatifs aux exercices réalisés, en cours et prévisionnels, sont issus du consensus d'analystes financiers réalisé par FactSet. 
Kim et Ritter, 1999 ; Cheng et McNamara, 2000 ; Lie et Lie, 2002 ; Liu et al., 2002 et 2007; Schreiner et Spremann, 2007 ; Harbula, 2009) ;

- l'identification de ratios de performance les plus pertinents pour sélectionner les sociétés les plus «comparables» au sein de chacun des groupes de pairs sectoriels initialement constitués (cf. Alford, 1992 ; Hermann et Richter, 2003 ; Dittmann et Weiner, 2005).

\section{Principaux enseignements issus des résultats de l'étude empirique}

Notre étude s'intéresse plus particulièrement aux multiples de valeur d'entreprise (VE) comptant parmi les plus usuels et fondés sur des indicateurs comptables ou financiers relatifs aux exercices réalisé, en cours ou prévisionnel (VE/capitaux employés, VE/chiffres d'affaires, VE/Ebitda et $\mathrm{VE} /$ Ebit). Les tableaux 3 et 4 ci-après présentent les dix multiples de valorisation retenus, avec leur notation ainsi que les statistiques descriptives et les mesures de corrélation correspondant à l'échantillon. Le tableau 5 et le graphique 2 présentent les statistiques descriptives des TEA de l'évaluation des différents multiples calculés ainsi que leur comparaison par référence à leur rang déterminé selon ces statistiques (écart interquartile, médiane, écart type et moyenne des TEA de l'échantillon, nombre de TEA inférieur à $15 \%$ ).

Les principaux résultats empiriques de l'étude, validés statistiquement par la comparaison des TEA de l'évaluation des différents multiples calculés (cf. tableau 6), sont les suivants :

1) nous confirmons les principaux résultats des études empiriques publiées depuis la fin des années 1990 ; il ressort ainsi que la pertinence de la méthode des multiples est de nature à être améliorée en privilégiant : - le recours à une moyenne harmonique pour le calcul des multiples synthétiques comparativement à une moyenne arithmétique,

Tableau 3 - Multiples de valorisation comparés

\begin{tabular}{|l|c|c|c|}
\hline \multicolumn{1}{|c|}{ Indicateurs } & $\begin{array}{c}\text { Exercice } \\
\text { réalisé }\end{array}$ & $\begin{array}{c}\text { Exercice } \\
\text { en cours }\end{array}$ & $\begin{array}{c}\text { Exercice } \\
\text { prévisionnel }\end{array}$ \\
\hline Multiple de capitaux employés (CE) & $\mathrm{VE} / \mathrm{CE}_{0}$ & - & - \\
\hline Multiples de chiffre d'affaires (CA) & $\mathrm{VE} / \mathrm{CA}_{0}$ & $\mathrm{VE} / \mathrm{CA}_{1}$ & $\mathrm{VE} / \mathrm{CA}_{2}$ \\
\hline Multiples d'Ebitda & $\mathrm{VE} / \mathrm{Ebit}_{0}$ & $\mathrm{VE} / \mathrm{Ebit}_{1}$ & $\mathrm{VE} / \mathrm{Ebit}_{2}$ \\
\hline Multiples d'Ebit & $\mathrm{VE} / \mathrm{Ebitda}_{0}$ & $\mathrm{VE} / \mathrm{Ebitda}_{1}$ & $\mathrm{VE} / \mathrm{Ebitda}_{2}$ \\
\hline
\end{tabular}

$\mathrm{VE}=$ valeur d'entreprise $=\mathrm{S}+\mathrm{D} ; \mathrm{S}=$ capitalisation boursière $; \mathrm{D}=$ endettement net de l'exercice réalisé (dettes financières à court et long terme - trésorerie + passifs hors exploitation - actifs hors exploitation); $\mathrm{CE}=$ capitaux employés de l'exercice réalisé (immobilisations et besoin en fonds de roulement d'exploitation); $\mathrm{CA}=$ chiffre d'affaires ; Ebitda = Earning Before Interest, Tax, Depreciation and Amortization ; Ebitda = Earning Before Interest and Tax ; $0=$ date de clôture de l'exercice réalisé « n-1»;1 = date de clôture de l'exercice en cours « $\mathrm{n} » ; 2$ date de clôture de l'exercice prévisionnel « $\mathrm{n}+1 »$ (source des données : FactSet). 


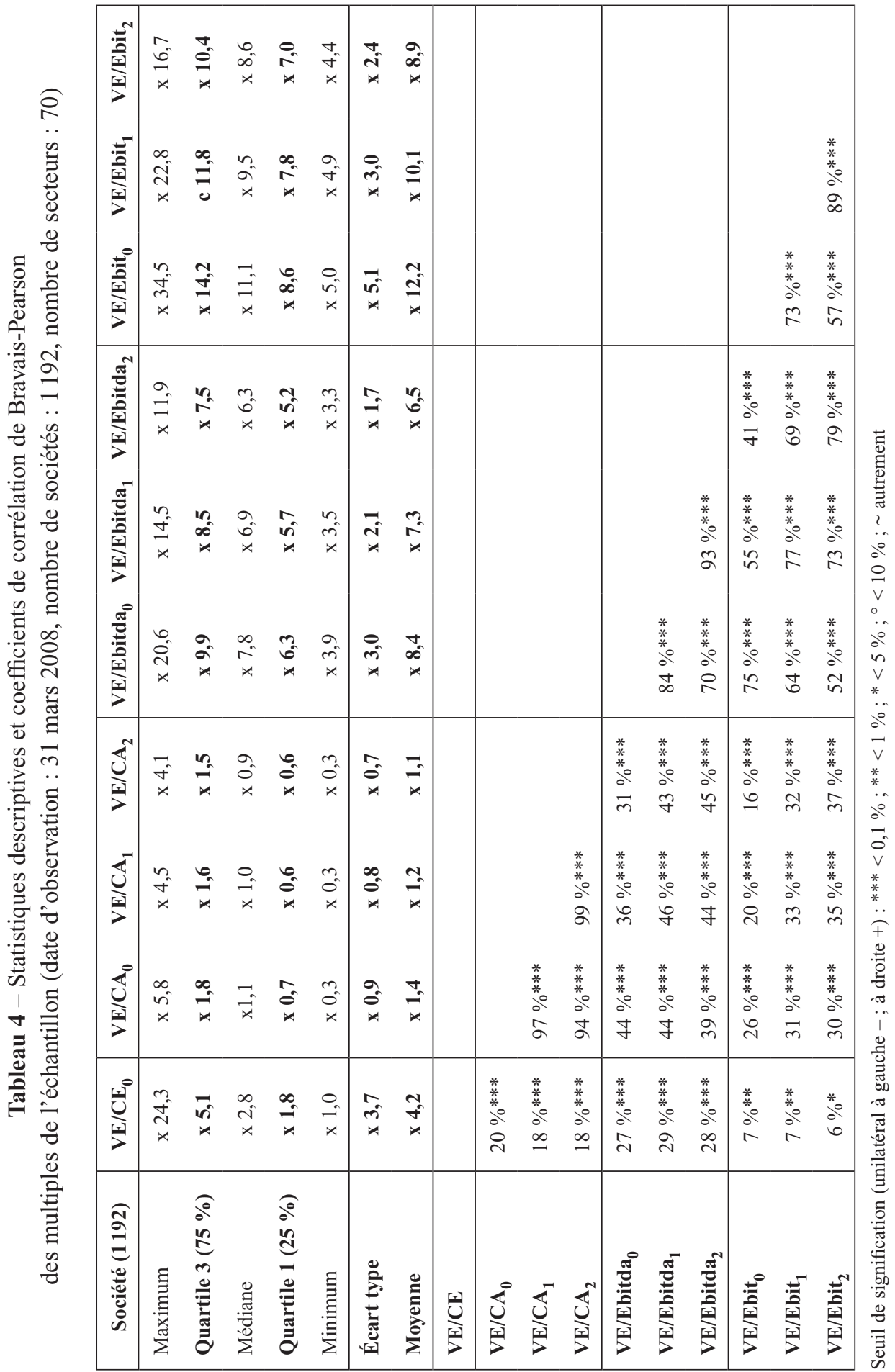




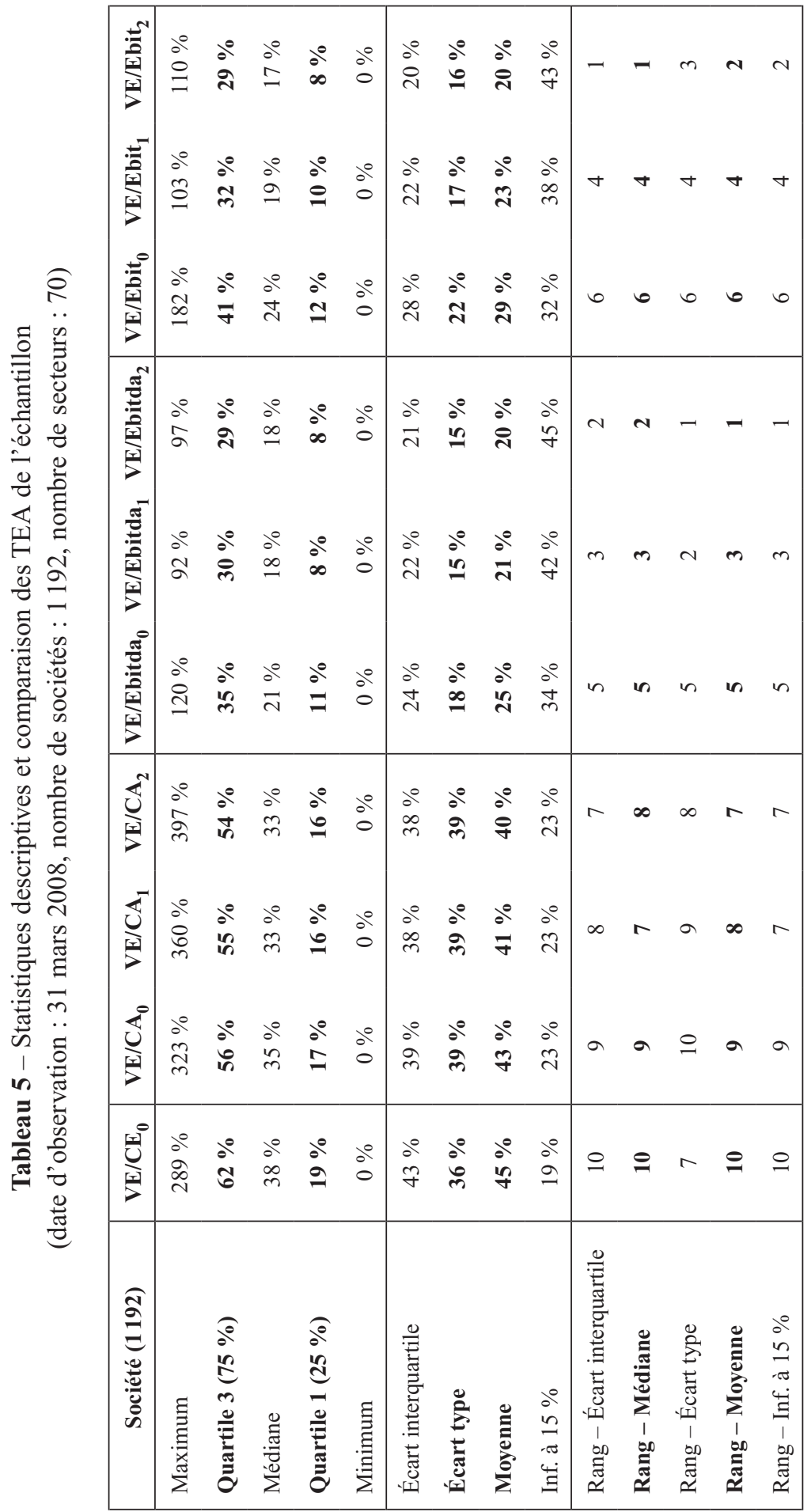


Graphique 1 - Comparaison de la moyenne, de la médiane et de la dispersion des TEA ${ }^{10}$ de l'échantillon (date d'observation : 31 mars 2008, nombre de sociétés : 1192 , nombre de secteurs : 70)
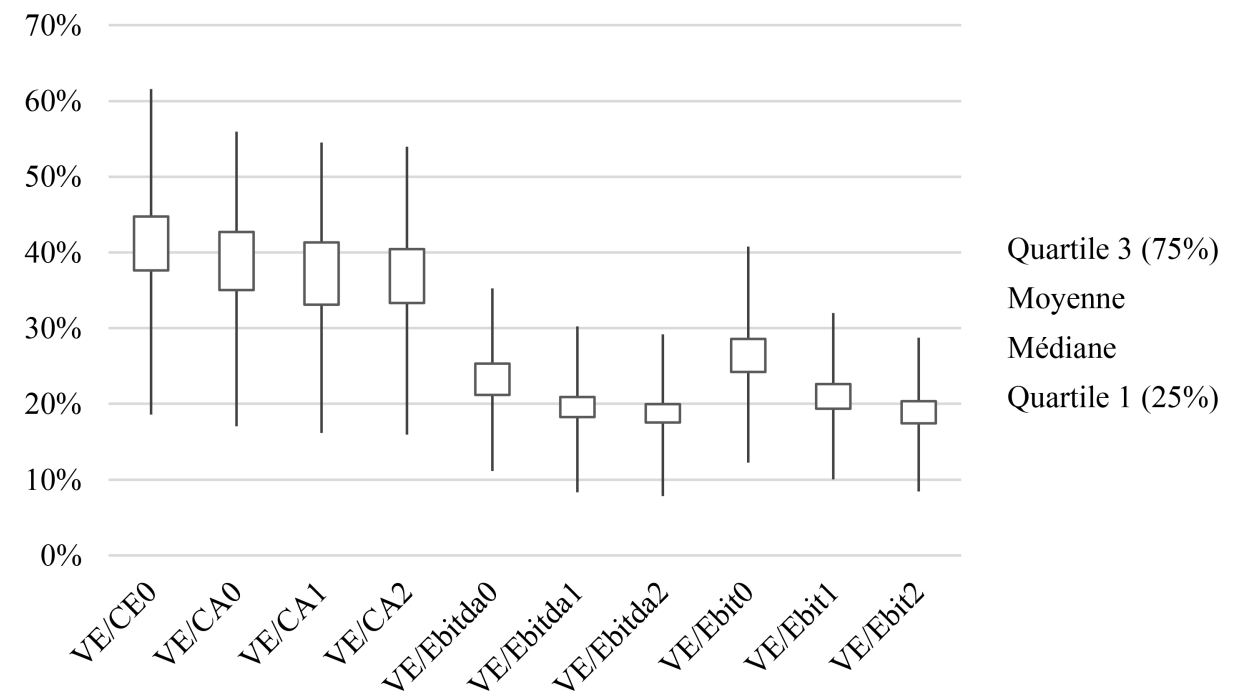

- le recours à des multiples de résultats (Ebitda ou Ebit) par comparaison à des multiples de chiffre d'affaires ou de capitaux employés,

- le recours à des multiples de résultats (Ebitda ou Ebit) relatifs à des exercices prospectifs (c'est-à-dire à l'exercice en cours ou, de préférence, à l'exercice prévisionnel $\mathrm{n}+1$ ) comparativement à des résultats relatifs à l'exercice réalisé ;

2) nous montrons également que le recours à des multiples d'Ebitda et d'Ebit est quasiment équivalent du point de vue de la pertinence de la méthode, quand il s'agit de résultats relatifs à l'exercice $n+1$, mais que la méthode est d'autant plus pertinente si l'on a recours à des multiples d'Ebitda comparativement à des multiples d'Ebit, quand il s'agit de résultats relatifs au dernier exercice publié ;

3) nous relevons enfin que l'utilisation de ratios de performance pour sélectionner les sociétés les plus comparables, au sein d'un groupe de pairs sectoriels, est de nature à améliorer la pertinence de la méthode dans le cas de certains multiples :

- le taux de rentabilité des capitaux employés pour les multiples de capitaux employés,

- le taux de marge d'exploitation sur chiffre d'affaires pour les multiples de chiffre d'affaires,

10. Dans ce graphique (et le suivant), le carré central correspond à l'écart entre moyenne et médiane. Il est gris quand la moyenne est supérieure à la médiane et noire dans le cas inverse. Les extrêmes correspondent aux bornes hautes du $1^{\text {er }}$ et du $3^{\mathrm{e}}$ quartile (soit la mesure de l'écart interquartile). 
Tableau 6 - Résultats des tests statistiques portant sur la pertinence comparée des multiples (dates d'observation : 31 mars 2006, 2007 et 2008)

\begin{tabular}{|c|c|c|c|}
\hline \multirow{2}{*}{$\begin{array}{l}\text { Critères de sélection } \\
\text { des multiples }\end{array}$} & \multicolumn{2}{|c|}{ Comparaison des TEA de l'évaluation } & \multirow{2}{*}{$\begin{array}{c}\text { Tests } \\
\text { de comparaison } \\
\text { des TEA } \\
\text { (seuils de } \\
\text { signification) }\end{array}$} \\
\hline & Pertinence $(+)$ & Pertinence (- ) & \\
\hline $\begin{array}{l}\text { Identification du mode } \\
\text { le plus pertinent pour } \\
\text { calculer les multiples } \\
\text { synthétiques }\end{array}$ & $\begin{array}{c}\text { Moyenne } \\
\text { harmonique } \\
\text { des multiples }\end{array}$ & $\begin{array}{c}\text { Moyenne } \\
\text { arithmétique des } \\
\text { multiples }\end{array}$ & $\mathrm{p}<0,1 \%$ \\
\hline \multirow{3}{*}{$\begin{array}{c}\text { Identification des } \\
\text { indicateurs comptables } \\
\text { ou financiers les } \\
\text { plus pertinents pour } \\
\text { calculer les multiples } \\
\text { de valorisation les plus } \\
\text { représentatifs }\end{array}$} & $\begin{array}{l}\text { VE/Résultats } \\
\text { (Ebitda et Ebit) }\end{array}$ & $\begin{array}{l}\mathrm{VE} / \mathrm{CE} \\
\mathrm{VE} / \mathrm{CA}\end{array}$ & $\mathrm{p}<0,1 \%$ \\
\hline & $\begin{array}{l}\text { VE/Résultats } \\
\text { prospectifs (n et } n+1) \\
\text { (Ebitda et Ebit) }\end{array}$ & $\begin{array}{l}\text { VE/Résultats réalisés } \\
\text { (Ebitda et Ebit) }\end{array}$ & $\mathrm{p}<0,1 \%$ \\
\hline & $\begin{array}{l}\text { Résultats réalisés } \\
\text { VE/Ebitda }\end{array}$ & $\begin{array}{c}\text { Résultats réalisés } \\
\text { VE/Ebit }\end{array}$ & $\mathrm{p}<0,1 \%$ \\
\hline \multirow{3}{*}{$\begin{array}{l}\text { Identification des } \\
\text { ratios de performance } \\
\text { les plus pertinents } \\
\text { pour sélectionner } \\
\text { les sociétés les plus } \\
\text { « comparables » }\end{array}$} & $\begin{array}{c}\mathrm{VE} / \mathrm{CE} \\
\text { Sélection } \\
\text { des comparables/ } \\
\text { secteur + taux } \\
\text { de rentabilité } \\
(\mathrm{r}=\text { Ebit } / \mathrm{CE})\end{array}$ & $\begin{array}{c}\mathrm{VE} / \mathrm{CE} \\
\text { Sélection des } \\
\text { comparables/secteur } \\
\text { seul }\end{array}$ & $\mathrm{p}<0,1 \%$ \\
\hline & $\begin{array}{c}\text { VE/CA } \\
\text { Sélection } \\
\text { des comparables/ } \\
\text { secteur }+ \text { taux } \\
\text { de marge } \\
(\mathrm{m}=\text { Ebit } / \mathrm{CA})\end{array}$ & $\begin{array}{c}\mathrm{VE} / \mathrm{CA} \\
\text { Sélection des } \\
\text { comparables/secteur } \\
\text { seul }\end{array}$ & $\mathrm{p}<0,1 \%$ \\
\hline & $\begin{array}{l}\text { VE/Résultats réalisés } \\
\text { (Ebitda et Ebit) } \\
\text { Sélection } \\
\text { des comparables/ } \\
\text { secteur + taux } \\
\text { de croissance attendu } \\
\text { (g = Résultats } \\
\text { prévisionnels/ } \\
\text { Résultats réalisés) }\end{array}$ & $\begin{array}{l}\text { VE/Résultats réalisés } \\
\text { (Ebitda et Ebit) } \\
\text { Sélection des } \\
\text { comparables/secteur } \\
\text { seul }\end{array}$ & $\mathrm{p}<0,1 \%$ \\
\hline
\end{tabular}


- le taux de croissance anticipé des résultats (Ebitda ou Ebit) pour les multiples de résultats relatifs à l'exercice réalisé.

\section{III - CAS D'APPLICATION ET DISCUSSION}

\section{Cas d'application}

Même s'ils sont validés statistiquement, les résultats de l'étude sont d'ordre général et ne doivent donc pas exclure, en pratique, une analyse au cas par cas. La méthodologie mise en œuvre qui consiste à comparer les TEA de l'évaluation des différents multiples peut être aisément reproduite par les praticiens au cas particulier de chacune de leurs évaluations pour identifier :

- tant les indicateurs comptables et financiers les plus pertinents pour sélectionner les multiples synthétiques les plus représen- tatifs du niveau de valorisation des sociétés constituant un groupe de pairs sectoriels ; - que les ratios de performance les plus pertinents pour sélectionner les sociétés les plus comparables au sein de ce groupe de pairs sectoriels.

La pertinence de la méthodologie proposée est illustrée au travers d'un cas pratique : le tableau 7 et le graphique 2 présentent, à titre d'exemple, le calcul des TEA de l'évaluation résultant de l'application de la méthode des multiples dans le cas d'un groupe de pairs sectoriels constitué de 10 entreprises européennes cotées et représentatives du secteur des «biens de consommation durables - appareils électroniques », identifiées selon la classification sectorielle de FactSet de niveau 2 (tableau 7).

L'exemple montre que les résultats généraux de l'étude relatifs à la sélection des

Graphique 2 - Comparaison de la moyenne, de la médiane et de la dispersion des TEA du groupe de pairs sectoriels (date d'observation : 31 mars 2008, nombre de sociétés : 10)

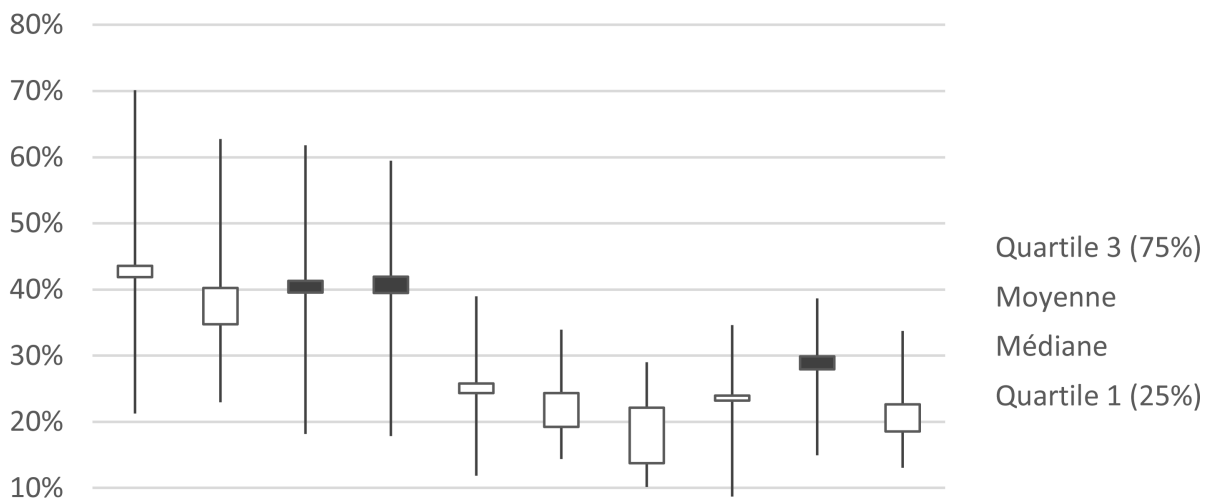

$0 \%$

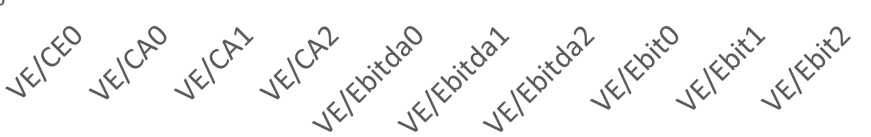




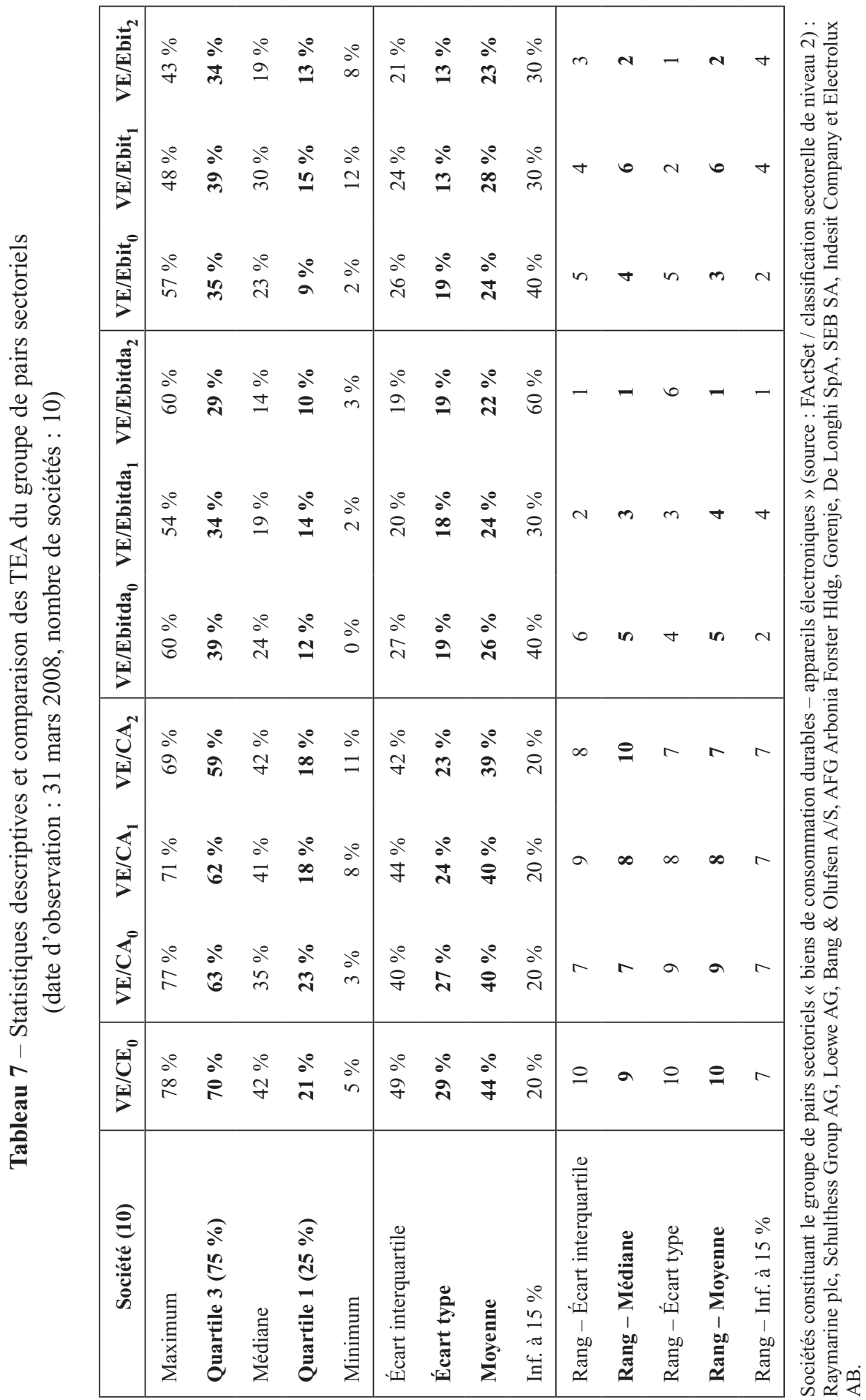


multiples les plus pertinents ne sont que partiellement confirmés dans le cas du secteur considéré, dans la mesure où le multiple d'Ebit relatif à l'exercice réalisé (VE/Ebit ${ }_{0}$ ) apparaît plus pertinent que le multiple d'Ebitda correspondant (VE/ Ebitda $_{0}$ ). Même si l'écart de performance entre ces deux indicateurs financiers est peu élevé dans ce cas particulier (soit un TEA moyen de $26 \%$ pour l'Ebitda contre $24 \%$ pour l'Ebit, et de $24 \%$ contre $23 \%$ en médiane $)^{11}$, il induit un questionnement sur l'impact du secteur quant à la pertinence des indicateurs comptable ou financiers du point de vue de l'évaluation.

La démarche mise en œuvre permet toutefois d'identifier les multiples les plus pertinents - dans le cas particulier du secteur étudié : si l'évaluateur a recours au multiple VE/Ebitda 2 , le plus pertinent, le TEA de son évaluation s'établit à $14 \%$ (selon la médiane des TEA), contre $24 \%$ en recourant au multiple VE/Ebitda 0 (22\% versus $26 \%$ selon la moyenne). Avec un TEA de $35 \%$ en médiane, il ressort que l'utilisation des multiples de chiffre d'affaires $\mathrm{VE} / \mathrm{CA}_{0}$ n'apparaît pas pertinente dès lors que les entreprises comparables ne sont pas sélectionnées, au sein de ce groupe de pairs sectoriel, à partir de leur taux de marge (mesuré par le rapport entre l'Ebit et le chiffre d'affaires). Une telle sélection viendrait réduire le TEA médian résultant de l'application de ce multiple à $23 \%$ contre $35 \%$.

\section{Discussion}

Sur un plan pratique, l'analyse du TEA permet d'améliorer le processus d'évaluation, tant pour identifier les multiples les plus pertinents que pour sélectionner les sociétés les plus comparables au sein d'un groupe de pairs sectoriels. Elle contribue, en outre, à améliorer la présentation et la justification des résultats obtenus par l'évaluateur dans tous les contextes d'évaluation (négociation, expertise indépendante, etc.).

Sur un plan académique, la méthodologie déployée permet d'envisager des recherches ultérieures complémentaires concernant : 1) la pertinence en valeur («value relevance ») des indicateurs comptables ou financiers du point de vue des investisseurs, avec des études plus à même d'orienter les régulateurs dans leur démarche de normalisation des éléments de communication financière des entreprises; 2) l'étude de l'impact du secteur sur la pertinence des différents indicateurs susceptibles d'être utilisés (Liu et al., 2002), ce y compris les facteurs explicatifs; 3) la sélection des sociétés les plus comparables au sein d'un groupe de pairs sectoriels en fonction d'autres ratios de performance (notamment la taille de l'entreprise, la liquidité de ses titres, son taux d'impôt effectif, etc.) ; 4) l'utilisation de multiples composites obtenus par régression linéaire compte tenu des ratios de performance des entreprises (taux de rentabilité, taux de croissance, etc.) (Bhojraj et Lee, 2002) ; enfin, 5) l'identification des multiples les plus pertinents dès lors qu'ils sont utilisés de manière combinée. Cette dernière question de recherche, qui n'a pas été abordée dans le cadre de cette étude, concerne le recours à une pondération possible des valeurs résultant

11. Il n'a pas été réalisé de tests statistiques sur la comparaison des moyennes, variances et proportions dans le cadre de ce cas d'application. 
de l'utilisation de différents multiples, de nature à améliorer la pertinence de la méthode (Cheng et McNamara, 2000 ; Liu et al., 2002 ; Yoo, 2006).

\section{CONCLUSION}

L'évaluation d'entreprise représente sans aucun doute un domaine d'application privilégié de la théorie financière (Lie et Lie, 2002). Elle constitue une étape préalable à toute décision d'acquisition ou de cession d'entreprises, que celles-ci soient cotées ou non (gestion de portefeuille, fusions \& acquisitions, capital développement), ainsi qu'une obligation lors des nombreuses opérations internes que sont amenés à réaliser les groupes, dans une perspective comptable (tests de dépréciation d'actifs par exemple), juridique (apports partiels d'actifs par exemple) ou fiscale (transferts d'actifs par exemple).

En vue d'apprécier la valeur de marché des entreprises, les professionnels ont recours à deux méthodes d'évaluation principales : la méthode des multiples, généralement issus de comparables boursiers, et la méthode des DCF fondée sur les prévisions de résultat du plan d'affaires.

Dans ce contexte, il convient de s'interroger sur la capacité des états financiers ou des analystes financiers à fournir l'information utile aux investisseurs pour guider leurs décisions. Il convient aussi de s'interroger sur le processus d'évaluation lui-même, ainsi que sur la capacité des évaluateurs à appliquer ces méthodes correctement en limitant les risques d'erreur, voire de manipulation des résultats.

Dans le cadre de la méthode des multiples, la question se pose de savoir si les évaluateurs sélectionnent les indicateurs de valeurs les plus pertinents ou les sociétés les plus comparables pour déterminer les multiples applicables à l'entreprise à évaluer ? Nous avons montré dans quelle mesure il est possible d'améliorer l'efficience du processus d'évaluation en se fondant sur les multiples qui minimisent le taux d'erreur absolu de l'évaluation.

En élargissant notre perspective, d'autres questionnements relatifs aux méthodes d'évaluation émergent. Dans la méthode des DCF, les évaluateurs mesurent-ils correctement le coût du capital utilisé pour actualiser les flux de trésorerie futurs ? Bancel et al. (2014) montrent qu'il n'existe pas de consensus entre les praticiens s'agissant des paramètres à prendre en compte pour appliquer la formule théorique du coût moyen pondéré du capital (CMPC). Les auteurs proposent une démarche permettant de mieux les appréhender afin d'améliorer la pratique.

La question se pose également quant à l'existence d'approches spécifiques à certains contextes, notamment en ce qui concerne l'évaluation des entreprises familiales, des entreprises coopératives ou des entreprises innovantes. Hirigoyen (2014), Sentis (2014) et Bessière et Stéphany (2014), respectivement, montrent dans quelle mesure la valeur de ces entreprises ne peut être appréciée sans prendre en compte leurs caractéristiques spécifiques, notamment la manière dont leurs actionnaires appréhendent leur performance dès lors que celle-ci n'est pas forcément reflétée par leurs états financiers. Une bonne raison de penser que les évaluateurs doivent nécessairement intégrer ces caractéristiques spécifiques afin d'améliorer l'efficience du processus d'évaluation, et cela quelle que soit la méthode mise en œuvre. 


\section{BIBLIOGRAPHIE}

Alford A.W. (1992). "The effect of the set of comparable firms on the accuracy of the priceearnings valuation method", Journal of Accounting Research, vol. 30, p. 94-108.

Bancel F., Mittoo X. (2013). "Théorie et pratique de l'évaluation financière », Revue Analyse Financière, ${ }^{\circ}$ 48, juillet-août-septembre, p. 80-83.

Bancel F., Lathuille Q., Lhuissier A. (2014). « De la difficulté de mesurer le coût du capital », Revue française de gestion, vol. 40, $\mathrm{n}^{\circ}$ 242, juin-juillet, p. 103-118.

Bellier Delienne A., Bour E. (2007). «Étude des méthodes d'évaluation des sociétés faisant l'objet d'une offre publique d'achat », La Revue du Financier, n ${ }^{\circ}$ 165, mai-juin, p. 4-31.

Bessière S., Stéphany E. (2014). «Le financement par crowdfunding : quelles spécificités pour l'évaluation des entreprises ? », Revue française de gestion, vol. 40, n²42, juinjuillet, p. 149-191.

Bhojraj S., Lee C.M.C. (2002). "Who is my peer? A valuation based approach to the selection of comparable firms", Journal of Accounting Research, vol. 40, n 2, May, p. 407-439.

Casta J.F. (2003). « La comptabilité en “juste valeur” permet-elle une meilleure représentation de l'entreprise », Juste valeur et évaluation des actifs financiers. Revue d'Économie Financière, $\mathrm{n}^{\circ} 71$, p. 11-24.

Chastenet E., Jeannin G. (2007). «Évaluation d'entreprise : les méthodes appliquées par les analystes financiers », Revue Analyse Financière, ${ }^{\circ}$ 24, p. 48-51.

Cheng C.S.A., McNamara R. (2000). "The valuation accuracy of the price-earnings and price-book benchmark valuation methods", Review of Quantitative Finance and Accounting, vol. 15, n 4, p. 349-370.

Courteau, L., Kao, J.L., O’Keefe, T., Richardson, G.D. (2006), "Relative accuracy and predictive ability of direct valuation methods, price to aggregate earnings method and a hybrid approach", Accounting \& Finance, vol. 46, n 4, p. 553-575.

Crow M.R., Gibbs A.K., Harms T.W. (2001). Valuation for Impairment Testing - The Finance and Accounting Professional's Guide to Valuing Reporting Units for Compliance with SFAS 142, Mercer Capital, Peabody Publishing, LP.

Damodaran A. (2006). "Valuation approaches and metrics: a survey of the theory and evidence", Working Paper, Stern School of Business.

DeAngelo L.E. (1990). "Equity valuation and corporate control", Accounting Review, vol. $65, \mathrm{n}^{\circ} 1$, January, p. 93-112.

Dittmann I., Weiner Ch. (2005). "Selecting comparables for the valuation of european firms", Working Paper, Humboldt-Universität zu Berlin.

Esty B.C. (2000). "What determines comparability when valuing firms with multiples?", Journal of Financial Education, ${ }^{\circ}$ 2, Fall, p. 24-33.

Harbula P. (2009). "Valuation multiples: Accuracy and drivers evidence from the european stock market", Business Valuation Review, vol. 28, n 4, p. 186-200. 
Herrmann V., Richter F. (2003), "Pricing with performance-controlled multiples", Schmalenbach Business Review, vol. 55, n³ July, p. 194-219.

Hirigoyen G. (2014), «Valeur et évaluation des entreprises familiales », Revue française de gestion, vol. 40, $\mathrm{n}^{\circ}$ 242, juin-juillet, p. 119-134.

Holthausen R.W., Zmijewski M.E. (2012). "Valuation with market multiples: How to avoid pitfalls when identifying and using comparable companies", Journal of Applied Corporate Finance, vol. 24, n 3, Summer, p. 26-38.

Kim M., Ritter J.R., (1999). "Valuing IPOs", Journal of Financial Economics, vol. 53, n 3, September, p. 409-437.

Kothari S.P., Ramanna K., Skinner D.J. (2010). "Implications for GAAP from an analysis of positive research in accounting", Journal of Accounting and Economics, vol. 50, $\mathrm{n}^{\circ} 2$, p. 246-286.

Lie E., Lie H.J. (2002). "Multiples used to estimate corporate value", Financial Analysts Journal, vol. 58, $\mathrm{n}^{\circ}$ 2, March-April, p. 44-54.

Liu J., Nissim D., Thomas J.K. (2002). "Equity valuation using multiples", Journal of Accounting Research, vol. 40, $\mathrm{n}^{\circ} 1, \mathrm{p} .135-172$.

Liu J., Nissim D., Thomas J.K. (2007), “Is cash flow king in valuations?”, Financial Analysts Journal, vol. 63, $\mathrm{n}^{\circ}$ 2, p. 1-23.

Meitner M. (2006). The market approach to comparable company valuation, Physica-Verlag.

Nobes C. (2006). "The survival of international differences under IFRS: towards a research agenda", Accounting and Business Research, vol. 36, n 3, p. 233-245.

Palepu, K.G., Healy P.M., Bernard V.L. (2000). Business Analysis and Valuation Using Financial Statements, $2^{\text {nd }}$ edition, South-Western.

Penman S.H. (2004). Financial Statement Analysis and Security Valuation, $2^{\text {nd }}$ edition, McGraw-Hill, New York, NY.

Penman S.H. (2006). "Handling valuation models", Journal of Applied Corporate Finance, vol. $18, \mathrm{n}^{\circ} 2$, p. $48-55$.

Schreiner A., Spremann K. (2007). "Multiples and their valuation accuracy in European equity markets", Working Paper, available at SSRN 957352.

Sentis P. (2014). "L'évaluation financière des coopératives modernes », Revue française de gestion, vol. 40, $\mathrm{n}^{\circ}$ 242, juin-juillet, p. 135-148.

Suozzo P., Cooper S., Sutherland G., Deng Z. (2001). "Valuation multiples: A primer", Working Paper, UBS Investment Bank.

Yoo Y.K. (2006). "The valuation accuracy of equity valuation using a combination of multiples", Review of Accounting and Finance, vol. 5, n² 2, p. 108-123. 
